\title{
Meningioma de la vaina del nervio óptico y tratamiento con radioterapia conformacional. Reporte de un caso clínico tratado con largo seguimiento Optic nerve sheath meningioma treated with radiation conformal therapy. Clinical case report with long follow up
}

Gustavo Zomosa R ${ }^{1}$, Sebastián Cruz T. ${ }^{2}$, Gonzalo Miranda G. ${ }^{3}$, Hans Harbst S. ${ }^{4}$

1 Neurocirujano. Servicio de Neurocirugía, Hospital Clínico de la Universidad de Chile. Unidad de Neuro-oncología. Santiago, Chile.

2 Interno Medicina. Facultad de Medicina Universidad de Chile. Santiago, Chile.

3 Neurorradiólogo. Servicio de Imagenología, Hospital Clínico de la Universidad de Chile. Unidad de Neuro-oncología. Santiago, Chile.

4 Radio-Oncólogo, Clínica Alemana de Santiago, Chile.

Rev. Chil. Neurocirugía 42: 133-136, 2016

\begin{abstract}
Resumen
Introducción: Los meningiomas de la vaina del nervio óptico (MVNO) son tumores inusuales de la vía visual anterior. Sin tratamiento, el crecimiento del tumor lleva a pérdida visual progresiva hasta la ceguera debido a la compresión que ejerce sobre el nervio óptico. Presentación del caso: Paciente, sexo femenino, 42 años, sin antecedentes mórbidos, que en 1992 , inicia cuadro de disminución de la agudeza visual del ojo izquierdo. La resonancia magnética (RM) cerebral informa una lesión expansiva fusiforme en relación a la vaina del nervio óptico izquierdo que lo engloba, altamente sugerente de MVNO. En esa ocasión se realiza exploración de órbita la cual resulta frustra, por lo que se decide observación con controles clínicos e imagenológicos anuales, permaneciendo estable. Aproximadamente diez años más tarde, en el lapso de un año, inicia agravación progresiva de la agudeza y campo visual, ptosis palpebral y alteraciones de la oculo-motilidad del ojo izquierdo, objetivadas por examen neuro-oftalmológico, por lo que se sospecha progresión de MVNO. Se solicita nueva RM que confirma crecimiento significativo del tumor. Se descarta nuevo abordaje quirúrgico por riesgo de mayor deterioro visual y se efectúa radioterapia conformacional con una dosis de 54 Gy. Actualmente, a la edad de 65 años, se cumplen 24 años de evolución, 13 post radioterapia, confirmándose estabilidad clínica y radiológica de MVNO. Discusión: La radioterapia, cuando se observa progresión de la enfermedad, ha demostrado ser una terapia eficaz para los MVNO, con menos complicaciones y con mejores resultados en la preservación de la función visual a largo plazo.
\end{abstract}

Palabras clave: Meningioma de la vaina del nervio óptico, radioterapia conformacional, radiocirugía.

\begin{abstract}
Introduction: Optic nerve sheath meningiomas (ONSM) are rare tumors of the anterior visual pathway. Without treatment, tumor growth leads to progressive loss of visual acuity and blindness due to optic nerve compression. Case report: Patient, female, 42 years without other morbility , begins in 1992 with decreased visual acuity of the left eye, magnetic resonance imaging (MRI) showed enlargement of the left optic nerve sheath, suggestive of ONSM. On that occasion, orbit exploration failed, so it was decided to follow up with annual clinical and imaging controls. About ten years later, begins with progressive deterioration of visual acuity and visual field, with ptosis and ocular motor palsy of the left eye, confirmed with neuro-ophtalmological examinations. MRI shows tumor progression. A new surgical approach was discarded by the risk of visual worsening. A conformal radiotherapy was performed with a fractionated 54 Gy dose. Today, at age 65, after 24 years of follow up,13 post radiation therapy. clinical and radiological stability of ONSM is confirmed. Discussion: Conformal radiotherapy has been
\end{abstract}


shown as an effective therapy, with fewer complications and better outcomes in the preservation of visual function in the long term follow up.

Key words: Optic sheath meningioma, Conformal Radiotherapy, Radiosurgery.

\section{Introducción}

Los meningiomas de la vaina del nervio óptico (MVNO) son tumores inusuales de la vía visual anterior, correspondiendo al $2 \%$ de los tumores orbitarios y del $1-2 \%$ del total de los meningiomas ${ }^{1,2,3,4}$. Sin tratamiento, el crecimiento del tumor conduce a la pérdida de la agudeza visual progresiva hasta la ceguera debido a la compresión que ejerce sobre el nervio óptico, sin necesariamente invadirlo ${ }^{5,6,7}$. Estos tumores pueden ser primarios, que se originan en las meninges de la vaina del nervio óptico intraorbitario o en las del canal óptico (intracanaliculares) o secundarios que surgen de las meninges circundantes, extraorbitarias, las del seno cavernoso, ligamento falciforme, procesos clinoídeos, ala esfenoidal o fosa pituitaria, que posteriormente crecen hacia la vaina del nervio óptico ${ }^{8}$. Histológicamente, los más frecuentes son los transicionales, con menor frecuencia se presentan los sinciciales. No se han descrito MVNO malignos ${ }^{9}$

La presentación clínica más frecuente del MVNO es la pérdida progresiva de la agudeza visual o campo visual, sin dolor, en una mujer de 40 ó 50 años, con una evolución que varía entre 1 a 5 años. Existe una tríada clásica de presentación clínica que consiste en pérdida progresiva de la visión, no dolorosa, con la presencia de shunt optociliares y atrofia óptica en el fondo de ojo. Los Shunts se consideran patognomónicos de los MVNO; sin embargo, sólo está presente en $30 \%$ de los casos $^{3}$. Otros hallazgos comunes en los casos de MVNO incluyen proptosis, quemosis, edema palpebral y alteraciones de la oculomotilidad $3,4,8,10,11$.

El hallazgo más frecuente del defecto de campo visual es la constricción periférica, pero también se han descrito aumento de la mancha ciega y defectos altitudinales ${ }^{9}$. La proptosis se presenta hasta en el $59 \%$ de pacientes y es de carácter leve, rara como síntoma inicial y es propio de lesiones canaliculares. También pueden presentarse diplopía y alteraciones de la mirada conjugada vertical $^{1}$, así como cefalea y dolor orbitario ${ }^{9}$.

\section{Presentación de caso}

Paciente, sexo femenino, 42 años (en 1992), sin antecedentes mórbidos de importancia, inicia cuadro de disminución de la agudeza visual del ojo izquierdo, sin alteración del campo visual, ni alteraciones de la oculomotilidad, motivo por el cual consultó en esa ocasión a oftalmólogo quien le solicitó resonancia magnética $(\mathrm{RM})$ que muestró una lesión expansiva fusiforme de la vaina del nervio óptico izquierdo y que margina al nervio óptico, altamente sugerente de meningioma de la vaina del nervio óptico (Figura 1). En esa ocasión se realizó una exploración de órbita con biopsia no concluyente, por lo que se decide seguimiento con controles clínicos e imagenológicos anuales. Aproximadamente diez años más tarde, (2002) a sus 52 años, consulta por deterioro progresivo, de aproximadamente un año de evolución, de agudeza y de campo visual, con ptosis palpebral y alteraciones de la oculomotilidad del ojo izquierdo. En este contexto se sospecha progresión del MVNO y es derivada al Servicio de Neurocirugía del Hospital Clínico de la Universidad de Chile. Se realizó una nueva RM que evidenció un significativo aumento de volumen de la lesión con respecto al examen inicial, marginando todo el trayecto orbitario del nervio óptico así como su porción canalicular, manteniendo sus características de señal y comportamiento con el uso de gadolinio (Figura 2). Se evalúa la posibilidad de nuevo abordaje quirúrgico, que se descarta debido al riesgo de agravar deterioro visual Por este motivo se discutió el caso en el Comité Neuro-oncológico y se opta por radioterapia conformacional 3D como la terapia más adecuada para su proceso expansivo; la que se realizó el año 2003 a sus 53 años. La paciente recibió con técnica isocéntrica una dosis de 54 Gy a 1,8 Gy por fracción. Se utilizaron 2 haces de fotones de $6 \mathrm{MV}$, uno coronal y otro lateral izquierdo. En el seguimiento la paciente evoluciona con mejoría parcial de su campo visual, estabilizándose el deterioro de la agudeza visual. Actualmente, a la edad de 65 años, se cumplen 13 años de seguimiento, post radioterapia y más de 24 años desde el inicio de los síntomas, confirmándose estabilidad clínica y radiológica del MVNO objetivada con RM donde no se evidencian cambios significativos de volumen ni señal (Figura 3 .

\section{Discusión}

El diagnóstico de MVNO se puede hacer por RM con imágenes características como el "tram track" en cortes axiales o "doughhut", aunque éstas también pueden verse en el pseudotumor, linfoma del nervio óptico y neuritis óptica $^{12}$. Debido a esto que el diagnóstico fundamentalmente se basa en cortes coronales de contraste con gadoli-

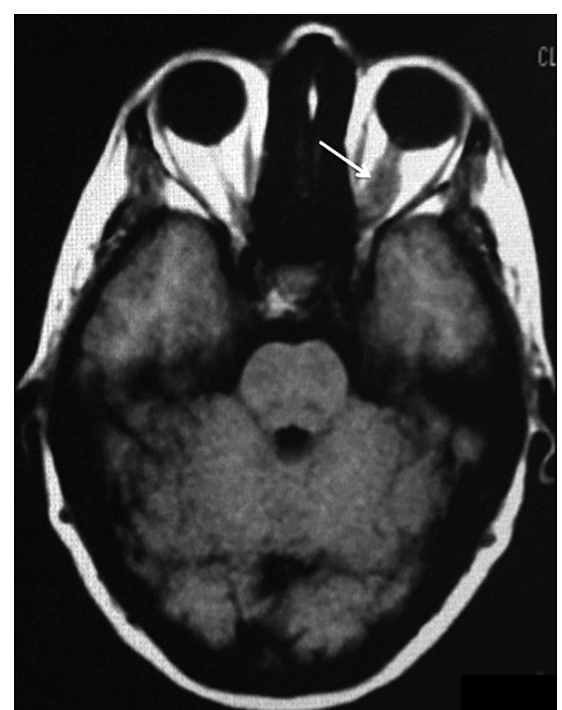

Figura 1. RM inicial en secuencia pondera en T1, que muestra una lesión expansiva de morfología fusiforme (Flecha blanca) en relación a la vaina del nervio óptico izquierdo. 


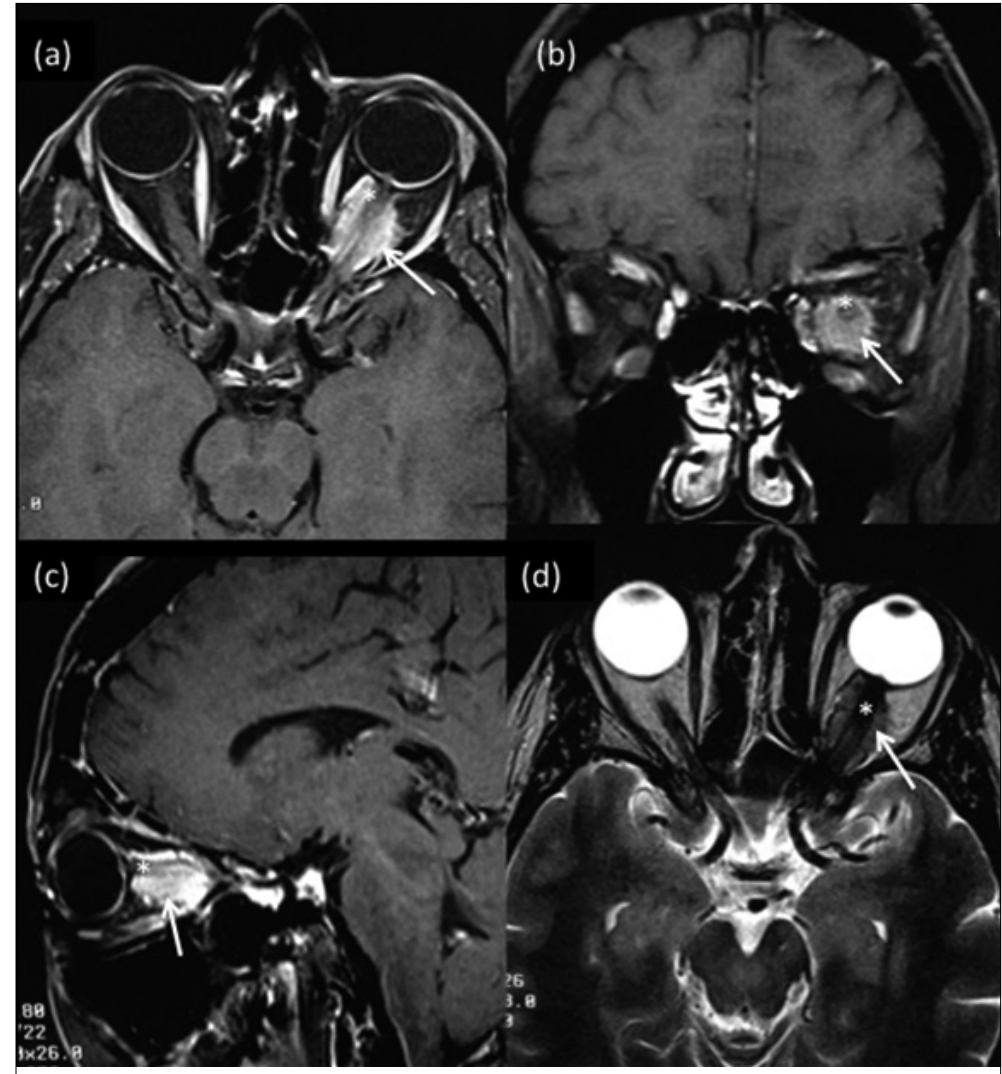

Figura 2. $R M$ en secuencias ponderadas $T 1$ con gadolinio fat sat en los planos axial (a), coronal (b) y sagital (C), y secuencia ponderada en T2 en el plano axial. Lesión expansiva fusiforme comprometiendo a la vaina del nervio óptico izquierdo (flecha blanca) con intenso realce con el uso de gadolinio y de iso a baja señal en secuencias T2. Es posible visualizar el nervio óptico en la porción central de esta masa (asterisco).

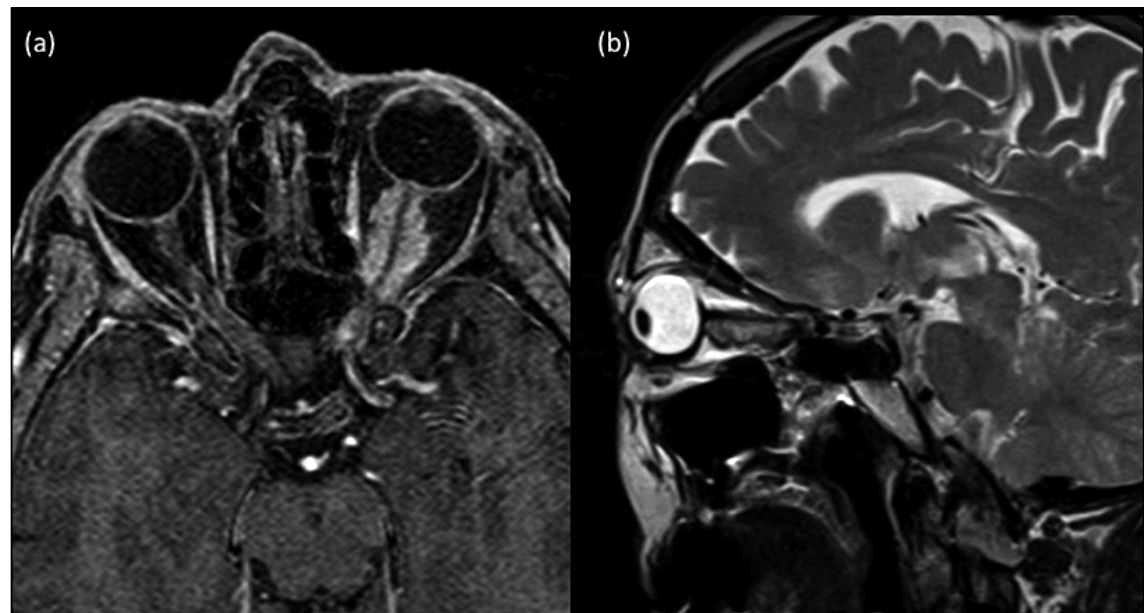

Figura 3. RM de control tomada 13 años después del tratamiento con radioterapia. Secuencia pondera en T1 gadolinio en plano axial y secuencia ponderada en T2 en el plano sagital. En comparación con los exámenes previos se evidencia estabilidad lesional sin cambios significativos en el volumen y señal de la lesión. nio de la secuencia T1 con supresión grasa, donde se aprecia el tumor, generalmente tubular, rodeando al nervio que no contrasta ${ }^{13}$. El diagnóstico de MVNO se realiza actualmente en base al cuadro clínico y los hallazgos neurorradiológicos, sin necesidad de biopsia, en la mayor parte de los casos ${ }^{9}$.

Es necesario conocer algo de la historia natural de este tumor para decidir su manejo, ya que la gravedad y el curso del déficit visual es variable, pero recientemente se sugiere que la mayoría de los pacientes con visión $20 / 50$, o mejor, podrían mantener el nivel visual por 5 años $^{12}$, pero globalmente el $85 \%$ van a perder visión durante su evolución ${ }^{1,12}$. Es por estos hechos que el tratamiento aún es controversial ${ }^{11}$.

De las alternativas de tratamiento existentes la primera es la observación, como se menciona previamente y se puede plantear en un grupo seleccionado de pacientes con visión útil y en tumores situados cerca del vértice orbitario, con estricto seguimiento clínico y con RM semestral. Si se evidencia deterioro clínico visual está indicada una terapia activa ${ }^{12}$. Los riesgos de ésta alternativa, se producen en los casos de MVNO intracanaliculares, ya que se ha reportado afectación del ojo contralateral en $38 \%$ de los casos, en ausencia de tratamiento, por lo que existe un riesgo sustancial de que ambos ojos se vean afectados ${ }^{6}$.

La cirugía, en una serie antigua de Dutton ${ }^{1}$, de 120 pacientes operados sólo un $5 \%$ mejorra su visión y el resto la pierde en el post-operatorio, lo que es ratificado recientemente por Turbin ${ }^{12}$. Se considera la biopsia-descompresión sólo en casos de visión no útil, con proptosis acentuada, extensión intracraneana o con crecimiento hacia el nervio contralateral, lo que es raro en tumores primarios, siendo más propio de los secundarios ${ }^{1,2,5,6}$. La agravación postoperatoria de la función visual, se debe a que existe íntima relación entre el tumor y la vascularización del nervio óptico ${ }^{12}$, ya que comparten los vasos piales, lo que explica los síntomas por isquemia ${ }^{14}$. La resección en block que incluye el nervio sólo se justifica con amaurosis ${ }^{2}$. Otro intento de tratamiento quirúrgico es la fenestración de la vaina del nervio óptico, que sólo en algunos casos produce un alivio transitorio por lo que no se recomienda su uso ${ }^{2,12}$.

Otras terapias, como la radioterapia han reportado estabilización visual e 
incluso mejoría en varios grupos de pacientes ${ }^{14,15}$. Turbin efectúa un estudio en que compara 4 grupos con distintos esquemas terapéuticos: observación, cirugía exclusiva, combinada con radioterapia y radioterapia como terapia exclusiva y concluye que ésta última es la que tiene mejores resultados a largo plazo para la función visual ${ }^{16}$. La radioterapia, que fue 2D en este estudio, muestra un $33 \%$ de complicaciones retinales y cerebrales. Sin embargo, el advenimiento de la radioterapia conformacional, la cuál en Chile estaba recién implementándose en la época que la paciente recibe el tratamiento, vino a reducir estos efectos secundarios.
El desarrollo tecnológico para la administración de radioterapia, ha permitido reducir la exposición a la radiación de tejido adyacente disminuyendo la radiotoxicidad y con esto las complicaciones, motivo por el cual la radioterapia se ha establecido como la terapia de elección del MVNO en los últimos años. Recientemente la radiocirugía estereotáxica hipofraccionada con Gamma Knife Perfection ha sido reportada como terapia de elección ${ }^{17,18 .}$

\section{Conclusiones}

La radioterapia ha demostrado ofrecer una terapia eficaz en el tratamiento de pacientes con MVNO, siendo actualmente la radiocirugía estereotáxica hipofraccionada la modalidad terapéutica que tiene menos complicaciones y mejores resultados con respecto a la preservación y mejoría de la función visual. La tecnología en los últimos años se ha desarrollado ampliamente en este tipo de intervenciones, lo que hace prever un futuro con cada vez mejores resultados visuales en pacientes con meningioma de la vaina del nervio óptico.

Recibido: 27 de mayo de 2016 Aceptado: 30 de junio de 2016

\section{Bibliografía}

1. Dutton JJ. Optic nerve sheath meningiomas. Surv Ophthalmol 1992; 37: 167-183.

2. Edderman C, Liu J. Optic nerve sheath meningioma: Current Diagnosis and Treatment. Neurosurg Focus 2007; $23(5)$ : E5.

3. James C. O'Brien MD. Ophtic nerve sheath meningioma. American academy of ophtalmology. Up to date: on February 5, 2015.

4. Yamashita J, Handa H, Iwaki K, et al: Recurrence of intracranial meningiomas, with special reference to radiotherapy. Surg Neurol 1980; 14: 33-40.

5. Alper MG. Management of primary optic nerve meningiomas. Current status-therapy in controversy. J Clin Neuroophthalmol 1981; 1 : 101-117.

6. Berman D, Miller NR. New concepts in the management of optic nerve sheath meningiomas. Ann Acad Med Singapore 2006; 35:168174.

7. Kim JW, Rizzo JF, Lessell S. Controversies in the management of optic nerve sheath meningiomas. Int Ophthalmol Clin 2005; 45:15-23.

8. Saeed P, Rootman J, Nugent RA, White VA, Mackenzie IR, Koornneef L. Optic nerve sheath meningiomas. Ophthalmology 2006; 110: 2019-2030.

9. Shapey J, Sabin HI, Danesh-Meyer HV, Kaye AH. Diagnosis and management of optic nerve sheath meningiomas. J Clin Neurosci. 2013 Aug;20(8):1045-56. doi: 10.1016/j.jocn.2013.03.008. Epub 2013 Jun 25.

10. Sibony PA, Krauss HR, Kennerdell JS, Maroon JC, Slamovits TL. Optic nerve sheath meningiomas. Clinical manifestations. Ophthalmology 1984; 91: 1313-1326.

11. Wright JE, McNab AA, McDonald WI. Primary optic nerve sheath meningioma. Br J Ophthalmol 1989; 73: 960-966.

12. Turbin RE, Pokorny K. Diagnosis and treatment of orbital optic nerve sheath meningioma. Cancer Control 2004; 11: 334-341.

13. Mafee MF,Goodwin J, Dorodi S. Optic nerve sheath meningiomas. Role of MR imaging. Radiol Clin N Am 1999; $37: 37-58$.

14. Bloch O, Sun M, Kaur G, Barani IJ, Parsa AT. Fractionated radiotherapy for optic nerve sheath meningiomas. J Clin Neurosci. 2012 Sep;19(9):1210-5. doi: 10.1016/j.jocn.2012.02.010. Epub 2012 Jun 22.

15. Carrasco JR, Penne RB. Optic nerve sheath meningiomas and advanced treatment options. Curr Opin Ophthalmol 2004; 15: 406-410.

16. Turbin RE, Thompson CR, Kennerdell JS, Cockerham KP, Kupersmith MJ. A long-term visual outcome comparison in patients with optic nerve sheath meningioma managed with observation, surgery, radiotherapy, or surgery and radiotherapy. Ophthalmology 2002; 109: 890-900.

17. Kwon Y, Bae JS, Kim JM, Lee do H, y otros. Visual changes alter Gamma Knife Surgery for optic nerve tumors. J Neurosurg (Suppl) 2005; 102: 143-146

18. Baumert BG, Villa S, Studer G, Mirimanoff RO, et al. Early improvements in vision after fractionated stereotactic radiotherapy for primary optic nerve sheath meningioma. Radiother Oncol. 2004; 72(2): 169-174.

\section{Correspondencia a:}

Dr.Gustavo Zomosa

Neurocirujano del Servicio de Neurocirugía, Hospital Clínico de la Universidad de Chile.

Unidad de Neuro-oncología. Santiago, Chile.

gzomosar@ hotmail.com 